\title{
Salivary Analytes in Patients of Oral Potentially Malignant Disorders and their Role in Early Detection of Oral Cancer: A Cross-sectional Study
}

\author{
Prutha B Rathod ${ }^{1}$, Ajay Bhoosreddy ${ }^{2}$, Chetan Bhadage ${ }^{3}$, Pragati Bramhe ${ }^{4}$, Prajakta Chaudhari ${ }^{5}$, Priyanka Giri ${ }^{6}$
}

\begin{abstract}
Background: The comprehensive salivary analysis had revealed an overall altered salivary composition in oral squamous cell carcinoma (OSCC) indicating a compromised oral environment in OSCC and suggesting salivary analysis as a new diagnostic tool for oral cancer. The mechanism behind these alterations is unknown; however, previous studies hypothesized that the tissue-abusive habits present in these patients contribute to saliva alterations.

Objectives: Thus, the present study aims to find how far this hypothesis stands true. In addition, the present study also aims to find whether these same salivary analytes are altered in oral potentially malignant disorders (oral leukoplakia, oral submucous fibrosis) and in case of positive results, these salivary analytes detected in precancerous stage could prove of great significance for early detection of OSCC.

Materials and methods: Unstimulated saliva samples were collected from 15 patients with tissue-abusive habits and oral potentially malignant disorders (OPMD) and 15 otherwise healthy individuals with tissue-abusive habits but without lesions and subjected to flame photometry for estimation of levels of $\mathrm{Na}, \mathrm{K}$, and $\mathrm{Ca}$.

Results: It revealed elevated levels of $\mathrm{Na}$ and $\mathrm{Ca}$ and lower concentration of $\mathrm{K}$ in OPMD patients compared to healthy individuals.

Conclusion: We conclude that an altered level of salivary analytes in OPMD similar to that found in OSCC is suggestive of saliva as an emerging diagnostic tool.

Keywords: Oral potentially malignant disorders, Oral squamous cell carcinoma, Saliva.

Journal of Contemporary Dentistry (2019): 10.5005/jp-journals-10031-1259
\end{abstract}

\section{INTRODUCTION}

Oral squamous cell carcinoma (OSCC) is the most common head and neck malignancy. It has a worldwide incidence of 300,000 new cases each year accounting for $2-4 \%$ of all new cancers. ${ }^{1,2}$ Despite numerous advances in the diagnostic aids, the survival rate for OSCC is quite low. ${ }^{3}$

In spite of all the accessibility of the oral cavity to direct examination, these malignancies are often not detected until the last stage. Early detection followed by appropriate treatment can increase cure rates by $80-90 \%$ and can improve the quality of life. ${ }^{4}$ However, currently there are no scientifically validated techniques for the early detection of OSCC. ${ }^{5,6}$ Early detection of oral potentially malignant disorders (OPMDs) is critical for prognosis and survival rates of OSCC. The literature suggests various methods for such detection; however, said methods are sensitive but not specific enough to detect premalignant lesions in patients at high risk of oral cancer. ${ }^{7}$ This suggests an imperative need for developing new diagnostic tools that would improve early detection. Various biomarkers are identified that could be useful for diagnosis of underlying diseases. Biomarker or biological marker is a naturally occurring molecule, gene, or characteristic by which a particular pathological or physiological process, disease, etc., can be identified. Identifying these biological markers in body fluids and utilizing them to predict the development of cancer at its early stage or in the precancerous stage would constitute a revolutionary diagnostic tool.

Body fluids include blood, saliva, mucus, urine, etc. Blood is the major component of body fluids but saliva being inexpensive,
${ }^{1-6}$ Department of Oral Medicine and Radiology, MGV's KBH Dental College and Hospital, Nashik, Maharashtra, India

Corresponding Author: Prutha B Rathod, Department of Oral Medicine and Radiology, MGV's KBH Dental College and Hospital, Nashik, Maharashtra, India, Phone: +91 9665721615, e-mail: prutha396@ gmail.com

How to cite this article: Rathod PB, Bhoosreddy A, Bhadage $C$, et al. Salivary Analytes in Patients of Oral Potentially Malignant Disorders and their Role in Early Detection of Oral Cancer: A Cross-sectional Study. J Contemp Dent 2019;9(2):85-88.

Source of support: Nil

Conflict of interest: None

noninvasive, and easy to handle wins over blood as a diagnostic fluid to monitor health and disease. For patients, the noninvasive technique used for saliva collection dramatically reduces anxiety, discomfort, and simplifies procurement of repeated samples for longitudinal monitoring over time. Saliva is a complex biological fluid composed of water and organic and inorganic substances. ${ }^{8}$ Salivary composition enables it to fulfill multiple functional tasks, as it is based both on components that originate in the oral and oropharyngeal mucosa and in the salivary glands themselves, as well as components that originate in the serum. ${ }^{9}$ Accordingly, salivary analysis enables the evaluation of both local and systemic alterations. Saliva plays a pivotal role in the pathogenesis of OSCC..$^{10,11}$ Comprehensive salivary electrolyte analysis revealed an overall altered salivary composition in OSCC patients. ${ }^{12,13}$ 
The mechanism behind these alterations is unknown; however, previous studies ${ }^{13}$ hypothesized that the tissue-abusive habits present in these patients contribute to saliva alterations. Thus, the present study aims to find how far these hypotheses stand true.

In addition, the present study also aims to find whether the salivary electrolytes altered in OSCC show similar changes in OPMD patients. In case of positive results, these salivary analytes altered in OPMD as well as in OSCC would prove of great significance for early detection of OSCC.

\section{A IM}

To compare the levels of $\mathrm{Na}, \mathrm{K}$, and $\mathrm{Ca}$ in saliva of patients diagnosed with OPMDs [oral submucous fibrosis (OSMF), leukoplakia] with tissue-abusive habit and otherwise healthy individuals with tissueabusive habit but without disorders.

\section{Objectives}

- To estimate levels of $\mathrm{Na}, \mathrm{K}$, and $\mathrm{Ca}$ in the saliva of patients with OPMD and with tissue-abusive habits-group I

- To estimate levels of $\mathrm{Na}, \mathrm{K}$, and $\mathrm{Ca}$ in the saliva of patients with tissue-abusive habits but without OPMD—group II

- To compare the levels of $\mathrm{Na}, \mathrm{K}$, and $\mathrm{Ca}$ in the saliva of group I and group II.

\section{Materials and Methods}

\section{Study Design}

This cross-sectional prospective in vivo study was carried out with selected samples meeting the inclusion criteria and allocated to two groups as follows:

Group I-Patients with OPMD and habit of tobacco and alcohol. Group II-Patients with habit of tobacco and alcohol but without OMPD.

\section{Study Setting}

The study was conducted after the approval was obtained from the institutional ethical committee. The study included 20 patients visiting the Department of Oral Medicine and Radiology of Dental Institute in Northern Maharashtra meeting the inclusion criteria (Figs 1 and 2). The verbal and written informed consent was taken from all participants before commencement of the study. The study period was for a month in June 2018.

\section{Patient Selection Criteria} Inclusion Criteria

- Age above 18 years

- Patients with habit of tobacco (smoked and smokeless) and alcohol and OPMD-confirmed histopathology

- Patients with habit of tobacco (smoked and smokeless) and alcohol but without any OPMDs

\section{Exclusion Criteria}

- Patients with any systemic diseases

- Patients with periodontitis

- Patients with history of any cancer

- Patients with OPMD without habit history

- Patients with history of any treatment for OPMD for less than 3 months

\section{Method of Data Collection}

\section{Saliva Sample Collection}

Whole unstimulated saliva was collected using standard methods. ${ }^{14}$ Briefly, samples were collected between 9 am and $12 \mathrm{am}$. Furthermore, all collections were performed at a fixed time of the day to minimize fluctuations related to the circadian rhythm in salivary secretion. Subjects were instructed to avoid eating, drinking, or using oral hygiene products for less than 1 hour before sample collection. They were initially instructed to seat quietly on the dental chair and then asked to rinse mouth before collection. During the collection, subjects were asked to swallow first, tilt their head forward, and expectorate all saliva into a $50 \mathrm{~mL}$ centrifuge tube without swallowing until the volume was $5 \mathrm{~mL}$ (Fig. 3). Following the collection, the saliva was immediately centrifuged at $800 \mathrm{~g}$ at $4^{\circ} \mathrm{C}$ for 10 minutes and the resulting supernatant was used for further biochemical analysis.

\section{Biochemical Analysis}

The biochemical analysis of samples collected was performed in institution Pharmacy College laboratory under the supervision of a guide. For estimation of $\mathrm{Na}, \mathrm{K}$, and Ca levels, the collected samples were subjected to flame photometry and the measurements were recorded.

\section{Statistical Analysis}

The statistical analysis was done using the SPCC software and the unpaired $t$-test applied at 18 degrees of freedom and $95 \%$ confidence level.

\section{Results}

The mean age in group I was 39 years, whereas in group II mean age was 26 years. Table 1 shows age range of patients in both groups (Figs 1 to 3 ).

Table 1: Agewise distribution of patients in group I and group II

\begin{tabular}{lll}
\hline Age range & Group I & Group II \\
\hline $18-25$ & 1 & 3 \\
$25-30$ & 2 & 5 \\
$30-35$ & 4 & 1 \\
Above 35 & 3 & 1 \\
\hline
\end{tabular}

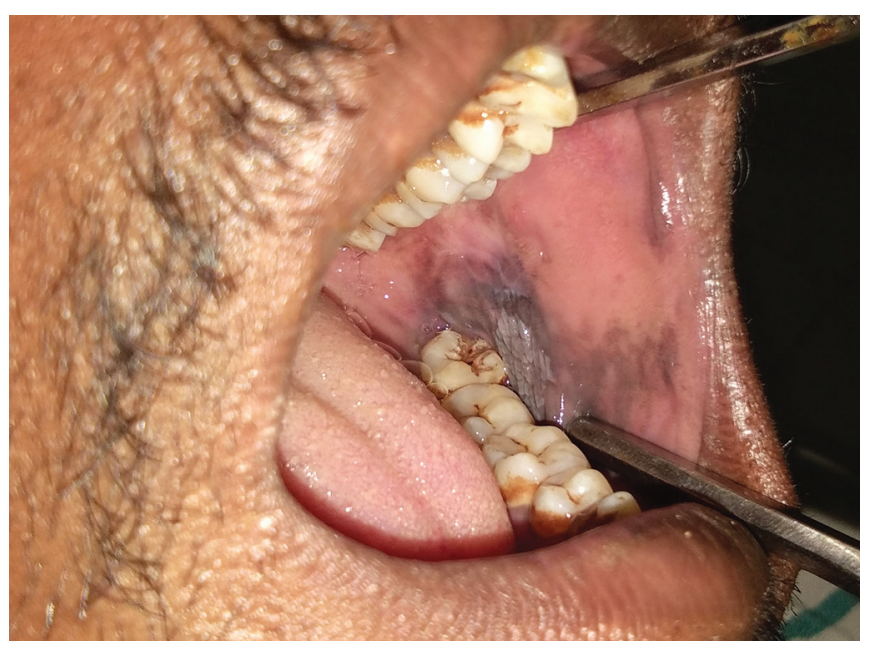

Fig. 1: Oral leukoplakia 
The OPMD included in group I showed mainly two lesions of patients, i.e., OSMF and leukoplakia (Table 2). When male and female patients' distribution was assessed in both groups, it was found that in group I for OSMF patients it was 2:1 and for leukoplakia it was 3:1; in group II, there was equal distribution (1:1) as shown in Table 2.

When the $\mathrm{Na}, \mathrm{K}$, and Ca levels were evaluated in group I and group II, it showed that the mean score of Na levels was significantly higher ( $p=0.0001)$ and mean score of K levels was significantly lower $(p=0.0000)$ in group I when compared with group II. Though Ca levels were found slightly increased in group I, the difference was not significant $(p=0.0736)$ as shown in Table 3 and Figure 4.

\section{Discussion}

Oral squamous cell carcinoma is the sixth most common human cancer with increasing incidence. To control its growing incidence, diagnosis/monitoring of this disease is the need of the hour. In the present study, there is a wide range of difference in the mean age of two groups. The possible explanation for these variations could be the cumulative effect of tissue-abusive habits that leads to formation of oral lesions in later life. Shpitzer et al. ${ }^{12}$ reported that concentrations of sodium, calcium, phosphate, and magnesium

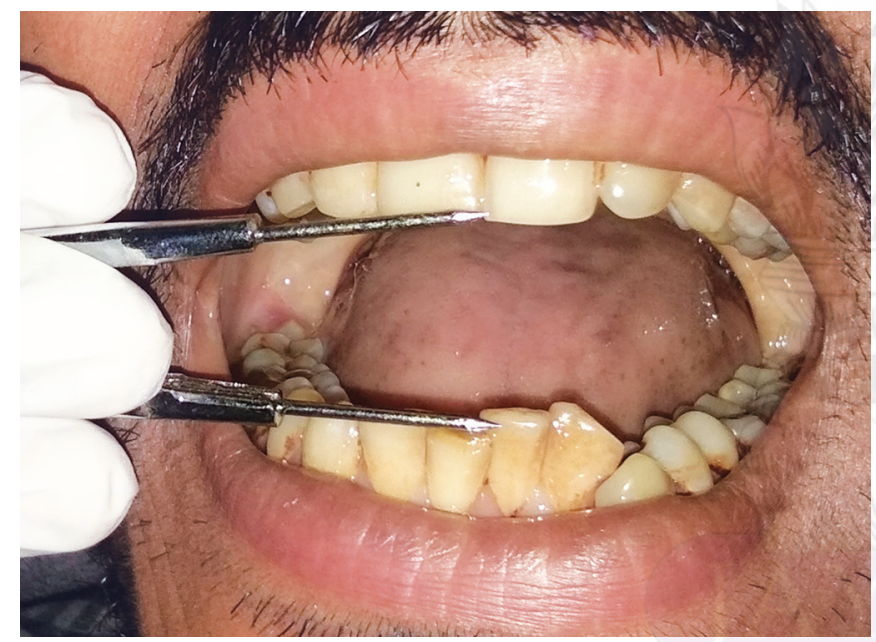

Fig. 2: Oral submucous fibrosis (OSMF)

Table 2: Patient's distribution in group I and group II

\begin{tabular}{llcl}
\hline & & Total & Male:female \\
\hline Group I & OSMF & 6 & $2: 1$ \\
& Leukoplakia & 4 & $3: 1$ \\
Group II & & 10 & $1: 1$ \\
\hline
\end{tabular}

were significantly higher in 25 patients with OSCC when compared to the 25 healthy controls whereas potassium concentrations were significantly lower in controls when compared to the OSCC patients. This is partially in concordance with our results as we also found increased levels of sodium and calcium and significantly lower levels of potassium in patients with OPMD when compared to the controls.

Zuabi et al. ${ }^{15}$ found that the subjects with periodontal disease had elevated concentrations of sodium, calcium, and magnesium; however, within this group smokers with periodontal disease had

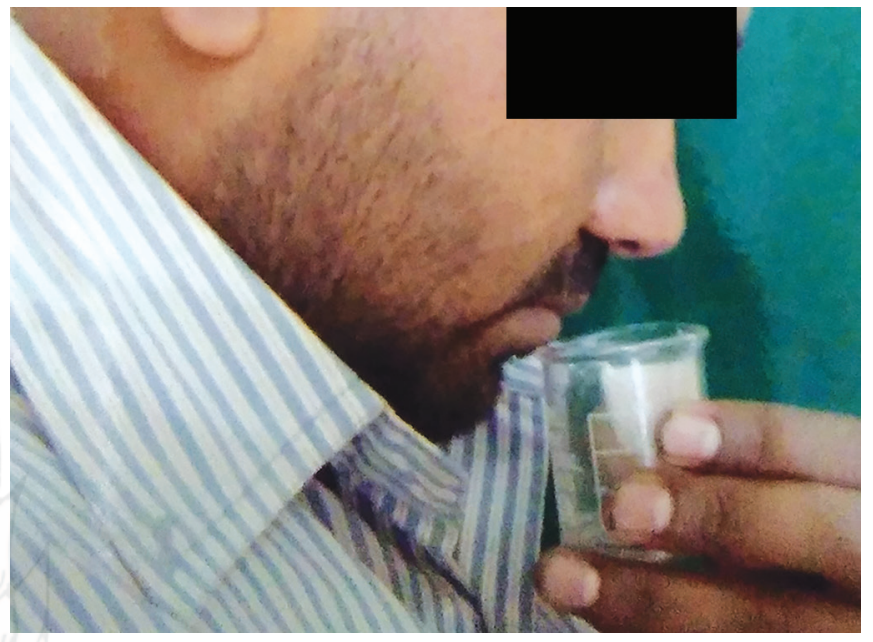

Fig. 3: Saliva sample collection

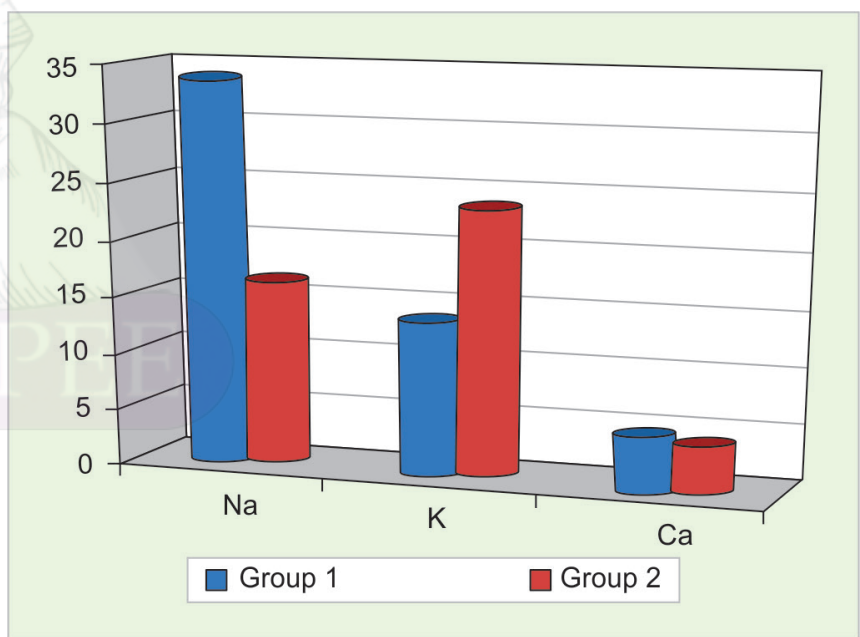

Fig. 4: Graphical presentation of difference in the levels of $\mathrm{Na}, \mathrm{K}$, and $\mathrm{Ca}$ among group I and group II

Table 3: Comparison of salivary analytes levels in group I and group II

\begin{tabular}{lllllll}
\hline Salivary analytes & Group & Mean score & SD & SE & t value & p value \\
\hline $\mathrm{Na}$ & Group I & 34 & 10.2956 & 3.4612 & 0.0001 (S) \\
& Group II & 16.88 & 3.7108 & & -4.758 & 0.0000 (S) \\
$\mathrm{K}$ & Group I & 13.8 & 3.2342 & 2.1401 & & \\
& Group II & 23.98 & 5.9431 & & 0.5149 & 0.0736 (NS) \\
$\mathrm{Ca}$ & Group I & 5 & 1.1269 & 0.4243 & \\
\end{tabular}

$\mathrm{Na}$, sodium; $\mathrm{K}$, potassium; $\mathrm{Ca}$, calcium; SD, standard deviation; $\mathrm{SE}$, standard error; $\mathrm{S}$, significant; $\mathrm{NS}$, not significant 
reduced sodium, calcium, and magnesium. This is in contrast with our finding as all the participants of our study were free of any periodontal disease. Thus, the salivary changes seen in OPMD patients were independent of the periodontal status. Also in the present study patients with OPMD were all smokers but had elevated levels of sodium and calcium when compared to the controls who were also smokers. Thus, it's difficult to comment whether smoking alone is responsible for salivary changes in OPMD patients.

Mandel ${ }^{16}$ reported elevated sodium levels in diabetes mellitus patients. On the contrary, Marder et al. ${ }^{17}$ could not confirm that finding. Ben-Aryeh ${ }^{18}$ noted an elevation in salivary sodium in digitalized patients in the unstimulated whole saliva. However, in the present study neither of our patients was diabetic nor was anyone taking digitalis, so increased levels of sodium and calcium in our study are probably due to some other cause.

Fuchs et al. ${ }^{13}$ had hypothesized that elevated sodium and chloride levels, which are usually seen in sera of dehydrated patients, are not so much connected to the oral carcinoma itself but more related to the dehydration status of oral carcinoma patients who were all alcoholic. Also alcohol and smoke are considered to be one of the leading causes of OSCC. However, this hypothesis was invalid in the present study as both the groups were having patients with habit of alcohol and smoking but the salivary changes were significant in OPMD patients. The other possible explanation given by Schpitzer et al. ${ }^{12}$ for salivary changes in OSCC is the evercontinuous and intimate contact between saliva and the mucosa (where this cancer evolves). This hypothesis also holds true for the present study where similar salivary changes were found in OPMD patients suggesting salivary analysis as a new diagnostic/ monitoring tool for OSCC. However, the exact mechanism behind these salivary changes and the correlation between OPMD and OSCC need to be further studied.

\section{Conclusion}

Saliva used as a diagnostic tool has a better patient compliance as it is noninvasive, easy to handle, cost-effective, and it also eliminates risks of contamination and infection. The salivary electrolytes that are reported to be raised in OSCC are also raised significantly in OPMD. However, their association in diagnosis of OSCC at early stage needs to be evaluated with further research.

\section{Limitations AND SCOPE}

There is need for further studies with larger sample size with sensitivity and specificity to validate saliva as a diagnostic tool for early detection of oral cancer.

\section{References}

1. Kantola S, Parikka M, Jokinen K, et al. Prognostic factors in tongue cancer-relative importance of demographic, clinical and histopathological factors. Br J Cancer 2000;83(5):614-619. DOI: 10.1054/bjoc.2000.1323.

2. Myers JN, Elkins T, Roberts D, et al. Squamous cell carcinoma of the tongue in young adults: increasing incidence and factors that predict treatment outcomes. Otolaryngol Head Neck Surg 2000;122(1):44-51. DOI: 10.1016/S0194-5998(00)70142-2.

3. Gualtero DF, Suarez A. Biomarkers in saliva for the detection of oral squamous cell carcinoma and their potential use for early diagnosis: a systematic review. 2016;74(3):170-177.

4. Bohle G. Oral cancer update 2012. Be ever vigilant in diagnosis and early detection. J Okla Dent Assoc 2012;103(6):34-36.

5. Jemal A, Bray F, Center MM, et al. Global cancer statistics. CA Cancer J Clin 2011;61(2):69-90. DOI: 10.3322/caac.20107.

6. Mishra R. Biomarkers of oral premalignant epithelial lesions for clinical application. Oral Oncol 2012;48(7):578-584. DOI: 10.1016/ j.oraloncology.2012.01.017.

7. Mehrotra R, Gupta DK. Exciting new advances in oral cancer diagnosis: avenues to early detection. Head Neck Oncol 2011;3:33. DOI: 10.1186/1758-3284-3-33.

8. Chiappin S, Antonelli G, Gatti R, et al. Saliva specimen: a new laboratory tool for diagnostic and basic investigation. Clin Chim Acta 2007;383(1-2):30. DOI: 10.1016/j.cca.2007.04.011.

9. Nagler RM, Klein I, Zarzhevsky N, et al. Characterization of the differentiated antioxidant profile of human saliva. Free Radic Biol Med 32(3):268-277. DOI: 10.1016/S0891-5849(01)00806-1.

10. Reznick AZ, Hershkovich O, Nagler RM. Saliva-a pivotal player in the pathogenesis of oropharyngeal cancer. Br J Cancer 2004;91(1):111-118. DOI: 10.1038/sj.bjc.6601869.

11. Wu HJ, Chi CW, Liu TY. Effects of pH on nicotine-induced DNA damage and oxidative stress. J Toxicol Environ Health A 2005;68(17-18): 1511-1523. DOI: 10.1080/15287390590967478.

12. Shpitzer T, Bahar G, Feinmesser R, et al. A comprehensive salivary analysis for oral cancer diagnosis. J Cancer Res Clin Oncol 2007;133(9):613-617. DOI: 10.1007/s00432-007-0207-z.

13. Fuchs PN, Rogi D, Vidovi D, et al. Salivary Analytes in Patients with Oral Squamous Cell Carcinoma. Coll Antropol 2011;35(2):359-362.

14. Navazesh M. Methods for collecting saliva. Ann N Y Acad Sci 1993;694:72-77. DOI: 10.1111/j.1749-6632.1993.tb18343.x.

15. Zuabi O, Machtei EE, Ben-Aryeh $\mathrm{H}$, et al. The Effect of Smoking and Periodontal Treatment on Salivary Composition in Patients With Established Periodontitis. J Periodontol. 1999;70(10):1240-1246. DOI: 10.1902/jop.1999.70.10.1240.

16. Mandel ID. Sialochemistry in Diseases and Clinical Situations Affecting Salivary Glands. Crit Rev Clin Lab Sci 1980;12(4):321. DOI: 10.3109/10408368009108733.

17. Marder MZ, Abelson DC, Mandel ID. Salivary Alterations in Diabetes Mellitus. J Periodontol. 1975;46(9):567. DOI: 10.1902/jop.1975.46.9.567.

18. Ben-Aryeh H, Krivoy N, Makhoul N, et al. The correlation between serum digoxin and electrolytes in saliva and erythrocytes in digitalized patients. J Oral Med 1982;37(4):124. 